\title{
The scheme of wind-storage combined system capacity configuration based on random fuzzy chance constrained bi-level programming
}

\author{
Mingyong OU ${ }^{1}$, Zhenyu WU', a, Haifeng YU11, Xing JIANG ${ }^{1}$, Yinyi $L^{2}$ and Wenlin $L U^{1}$ \\ ${ }^{1}$ State Grid Hunan Electric Power Company Economic and Technological Research Institute, Changsha 410004, China \\ ${ }^{2}$ Changsha University of Science and Technology, Changsha 410114, China
}

\begin{abstract}
A random fuzzy chance constrained bilevel programming scheme for distributed wind-storage combined system is proposed. The random fuzzy simulation is used to describe the uncertainty of distributed wind power output. The reliability of randomness and ambiguity is taken as the index to evaluate the capacity allocation scheme of the distributed wind-storage combined system. Considering system power balance, opportunity measurement constraint of static security index and active management (AM) measures, the random fuzzy expectation value of maximum annual profit is set as the upper optimization goal, and the minimum random fuzzy expectation value of the distributed wind power active reduction is set as the lower optimization target. The scheme is constructed by judging whether the static security index of the upper goal satisfies the confidence level of the random fuzzy chance constraint and the coordination of the upper and lower goals. Finally, the random fuzzy simulation, the forward pushback power flow calculation and the genetic algorithm (GA) are applied to solve the model. The simulation result of IEEE 14-bus example shows the effectiveness and superiority of the model and scheme.
\end{abstract}

\section{Introduction}

With a flexible power regulation ability, energy storage equipments can greatly improve the access characteristics of wind turbines. However, the existing schemes of grid configuration can not be applied to the system with wind energy storage system. It is urgent to establish a grid configuration scheme considering the characteristics of wind turbine and energy storage.

Nowadays, the researches on the configuration of distributed wind power and energy storage are usually separate. $\mathrm{L}$. Li et al. propose a multi-stage programming modeland discretize and couple DG output and load data [1]. On the basis of considering wind power and load, A. Soroudi et al. add electricity price factor to construct different stages [2]. Based on chance constrained programming, the randomness of wind power and load is considered for reactive compensation configuration and distributed wind power location by S. Zhang, et al [3]. Based on fuzzy mathematics theory, the random fuzzy variables can be applied to obtain more uncertain information in configuration [4-5]. At present, there is no reserches of wind-storage combined system configuration based on random fuzzy chance constrained bilevel programming.

Generally, the paper is organized as follows: i) A random fuzzy chance constrained bilevel programming scheme for combined wind-storage system is proposed. ii) Presenting a method Combined with the random fuzzy simulation, the forward pushback power flow calculation and the genetic algorithm (GA) to solve the model. iii) Showing the the effectiveness and superiority of the model and algorithm with simulation of IEEE 14-bus example.

\section{Random fuzzy modeling of distributed wind-storage combined system output and static security}

\subsection{The sequential random fuzzy model of distributed wind power output.}

In the work, we choose some typical days form four seasons and divide each day into 24 hours to conduct random fuzzy simulation of wind power output respectively; The shape parameter $k$ in Weibull distribution is represented by triangular fuzzy variable $\xi_{k}=\left(\xi_{k}^{1}, \xi_{k}^{2}, \xi_{k}^{3}\right)$, the scale parameter $c$ by trapezoidal fuzzy variable $\xi_{c}=\left(\xi_{c}^{1}, \xi_{c}^{2}, \xi_{c}^{3}, \xi_{c}^{4}\right) ;$ The membership function can be described as follows:

$$
u(k)= \begin{cases}\left(k-\xi_{k}^{1}\right) /\left(\xi_{k}^{2}-\xi_{k}^{1}\right) & \xi_{k}^{1} \leq k<\xi_{k}^{2} \\ \left(\xi_{k}^{3}-k\right) /\left(\xi_{k}^{3}-\xi_{k}^{2}\right) & \xi_{k}^{2} \leq k<\xi_{k}^{3} \\ 0 & \text { else }\end{cases}
$$

\footnotetext{
a Corresponding author: 835570133@qq.com
} 


$$
u(c)= \begin{cases}\left(c-\xi_{c}^{1}\right) /\left(\xi_{c}^{2}-\xi_{c}^{1}\right) & \xi_{c}^{1} \leq c<\xi_{c}^{2} \\ 1 & \xi_{c}^{2} \leq c<\xi_{c}^{3} \\ \left(\xi_{c}^{4}-c\right) /\left(\xi_{c}^{4}-\xi_{c}^{3}\right) & \xi_{c}^{3} \leq c<\xi_{c}^{4} \\ 0 & \text { else }\end{cases}
$$

Wind speed is represented by random fuzzy variable $\xi_{v}$. Its chance-constrained distribution function of weibull distribution can be described as bellow:

$$
F\left(\xi_{v}\right)=\operatorname{ch}\left(v<\xi_{v}\right)=1-\exp \left[-\left(\xi_{v} / \xi_{c}\right)^{\xi_{k}}\right]
$$

The random fuzzy wind speed value as follows is obtained by inverse transformation of Eq. (4):

$$
v=c[-\operatorname{In}(1-F(v))]^{\frac{1}{k}}
$$

The probability of the wind speed above is equal to the probability of the current $k$ and $c$, which is marked as $\operatorname{Pos}\left\{\theta_{k c}\right\}$. According to the uncertainty theory, $\operatorname{Pos}\left\{\theta_{k c}\right\}=\operatorname{Pos}\left\{\theta_{k}\right\} \wedge \operatorname{Pos}\left\{\theta_{c}\right\}$, which is the smaller value in the membership of $k$ and $c$.

\subsection{Modeling of the wind-storage combined system}

Adjusting charging and discharging power in storage configuration to stabilize the fluctuation of wind power:

$$
P_{\mathrm{ess}, \mathrm{t}}^{\text {real }}=\left\{\begin{array}{cc}
P_{\mathrm{ess}, \mathrm{t}}+\left(P_{w, t}-P_{u p, t}\right) & P_{u p, t}<P_{w, t} \cup P_{u p, t}<P_{d o w n, t} \\
P_{\mathrm{ess}, \mathrm{t}} & P_{d o w n, t} \leq P_{u p, t} \leq P_{w, t}
\end{array}\right.
$$

where, $P_{\text {ess, } t}$ is the planned value of energy storage charging power for a given $t$-period, and the negative value represents discharge; $P_{w, t}$ represents wind power generated by random fuzzy simulation at t-time; $P_{u p, t}$ and $P_{\text {down, } t}$ are the maximum and minimum random fuzzy expectation values of wind power within a certain range of confidence in $\mathrm{t}$-period.

\subsection{Random fuzzy chance measure of static security index}

A method to solve the random fuzzy power flow of wind-storage combined system is proposed, in order to acquire the static security indexes such as node voltage, branch power and the limit of backward power. The specific steps are as follows:

1) Obtain the wind power output of each period by random fuzzy simulation, and simulate the load by adding random numbers of normal distribution on the basis of typical daily load data.
2) Calculate the power flow by the forward and backward substitution method.

3) Calculate the probability measure and its parameter membership function of the static security index which matches the static security range in the random simulated samples under each possibility. The expressions of the random fuzzy chance measure with node voltage, branch power and backward power in normal interval are as follows:

$$
\begin{aligned}
& \operatorname{Ch}\left\{U_{\text {min }} \leq \tilde{U} \leq U_{\text {max }}\right\}\left(\alpha_{u}\right)= \\
& \sup \left\{\beta_{u} \mid \operatorname{Cr}\left\{\theta \in \Theta \mid \operatorname{Pr}\left\{U_{\text {min }} \leq \tilde{U} \leq U_{\text {max }}\right\} \geq \beta_{u} \geq \alpha_{u}\right\}\right. \\
& \operatorname{Ch}\left\{\tilde{P}_{l} \leq P_{l \text { max }}\right\}\left(\alpha_{l}\right)= \\
& \quad \sup \left\{\beta_{l} \mid \operatorname{Cr}\left\{\theta \in \Theta \mid \operatorname{Pr}\left\{\tilde{P}_{l} \leq P_{l \text { max }}\right\} \geq \beta_{l}\right\} \geq \alpha_{l}\right\} \\
& \operatorname{Ch}\left\{\tilde{P}_{B} \geq 0\right\}\left(\alpha_{B}\right)= \\
& \quad \sup \left\{\beta_{B} \mid \operatorname{Cr}\left\{\theta \in \Theta \mid \operatorname{Pr}\left\{\tilde{P}_{B} \geq 0\right\} \geq \beta_{B}\right\} \geq \alpha_{B}\right\}
\end{aligned}
$$

where node voltage $\tilde{U}$, branch power $\tilde{P}_{l}$ and $\tilde{P}_{B}$ are random fuzzy variables; $\operatorname{Ch}\{\cdot\}(\alpha)$ is the chance for random fuzzy events; $\operatorname{Pr}$ is a probability measure; $\alpha_{\mathrm{u}}$, $\alpha_{\mathrm{l}}, \alpha_{\mathrm{B}}, \beta_{\mathrm{u}}, \beta_{l}, \beta_{\mathrm{B}}$ are the given confidence levels; $\left\{U_{\min } \leq \tilde{U} \leq U_{\max }\right\}$ is a random fuzzy event with node voltage in normal interval; $\left\{\tilde{P}_{l} \leq P_{l \max }\right\}$ is a random fuzzy event with branch power satisfying boundary conditions; And $\left\{\tilde{P}_{B} \geq 0\right\}$ is a random fuzzy event when the input power, sent by the low voltage side of distribution substation to the active distribution network, is greater than zero.

\section{The scheme of wind-storage combined system capacity configuration based on random fuzzy chance constrained bi- level programming}

\subsection{The upper-level optimization model}

\subsubsection{Objective function}

The upper-level optimization is to maximize the random fuzzy expected value of annual profit under the confidence level of chance measure. The objective function is as follows:

$$
\max \overline{f_{u}}
$$

\subsubsection{Constraint conditions}

Chance constraint conditions for objective functions can be described as:

$$
\operatorname{Ch}\left\{f\left(x, \xi_{k}\right) \geq \overline{f_{u}}\right\}(\gamma) \geq \delta
$$

and, 
$f\left(x, \xi_{k}\right)=C_{\mathrm{sal}(k)}+C_{\mathrm{net}(k)}+C_{\mathrm{e}(k)}-C_{\mathrm{AM}(k)}-C_{P \operatorname{loss}(k)}-C_{\mathrm{inv}}$

where, $x$ is a decision variable, representing the number of wind turbines connected; $\xi_{k}$ is the $k$ th random fuzzy variable; $\gamma$ and $\delta$ are the given confidence levels; $\overline{f_{u}}$ is the fuzzy expected value of annual profit under the confidence level of opportunity measure; $f\left(x, \xi_{k}\right)$ is the total annual profit of the $k$ th random fuzzy simulation; $C_{\mathrm{sal}(k)}$ is the annual sales income of the wind storage system; $C_{\text {net }(k)}$ is the annual sales income of traditional distribution network; $C_{\mathrm{e}(k)}$ is the annual income of environmental improvement; $C_{\mathrm{AM}(k)}$ is the annual fee of $\mathrm{AM} ; C_{P \operatorname{loss}(k)}$ is the annual expense of network loss and $C_{\text {inv( }(k)}$ is the annual investment cost of traditional distribution network. They are expressed as follows:

$$
\left\{\begin{array}{l}
C_{\mathrm{sal}(k)}=\sum_{m=1}^{4} N_{m} \sum_{n=1}^{24} \sum_{i=1}^{N_{\mathrm{DG}}}\left(P_{\mathrm{DG} i(k)}^{m, n}+P_{\mathrm{ess} i(k)}^{m, n}\right)\left(a_{i}-a_{i}^{\prime}\right) \\
C_{\mathrm{net}(k)}=\sum_{m=1}^{4} N_{m} \sum_{n=1}^{24}\left(P_{\mathrm{L}(k)}^{m, n}-\sum_{i=1}^{N_{\mathrm{DG}}}\left(P_{\mathrm{DG} i(k)}^{m, n}+P_{\mathrm{essi}(k)}^{m, n}\right)\right) E_{\mathrm{tra}} \\
C_{\mathrm{e}(k)}=\mathrm{I}_{\mathrm{e}} \sum_{m=1}^{4} N_{m} \sum_{n=1}^{24} \sum_{i=1}^{N_{\mathrm{DG}}}\left(P_{\mathrm{DG} i(k)}^{m, n}+P_{\mathrm{ess} i(k)}^{m, n}\right) \\
C_{\mathrm{AM}(k)}=\sum_{m=1}^{4} N_{m} \sum_{n=1}^{24} \sum_{i=1}^{N_{\mathrm{DG}}} c_{i, \mathrm{DG}}^{\mathrm{AM}}\left(P_{\mathrm{DG} i(k)}^{m, n}+P_{\mathrm{ess} i(k)}^{m, n}\right) \\
C_{P \operatorname{Loss}(k)}=\sum_{m=1}^{4} N_{m} \sum_{n=1}^{24} C_{\mathrm{Loss}} \Delta P_{\mathrm{Loss}(k)}^{m, n} \\
C_{\mathrm{inv}(k)}=(1+\eta)\left(\alpha_{\mathrm{DG}} \sum_{i=1}^{N_{\mathrm{DG}}} c_{D G, i} P_{N \mathrm{DG} i(k)}\right. \\
\left.\quad+\alpha_{\mathrm{ess}} \sum_{i=1}^{N_{\mathrm{ess}}} c_{e s s, i} P_{E S S i(k)}+\alpha_{\mathrm{f}} c_{\mathrm{f}}\right) \\
P_{N \mathrm{DG} i(k)}=X_{i \mathrm{DG}(k)} P_{N \mathrm{DG}}
\end{array}\right.
$$

where, $N_{m}$ is the number of days in each season; $N_{\mathrm{DG}}$ is the number of wind turbine nodes installed in the system; $P_{\mathrm{DG} i(k)}^{m, n}$ and $P_{\mathrm{ess} i(k)}^{m, n}$ are the wind power and the energy storage power on the nodes of the $n$th period in the $m$ season in the $k$ th random fuzzy simulation; $a_{i}$ and $a_{i}^{\prime}$ are the hourly electricity price factor and maintenance cost factor of the first node wind turbine; $P_{\mathrm{L}(k)}^{m, n}$ is the the total load of distribution network for each period in the $k$ th simulation; $E_{\text {tra }}$ is the profit per hour of the traditional energy unit; $I_{e}$ is the factor of environmental improvement; $c_{i, \mathrm{DG}}^{\mathrm{AM}}$ is the $\mathrm{AM}$ cost per unit generating capacity of the wind turbines installed at the selected node $i ; C_{\mathrm{Loss}}$ is the hourly loss cost; $\Delta P_{\operatorname{Loss}(k)}^{m, n}$ is the active power loss per hour of the $k$ th random fuzzy simulation; $\eta$ is the ratio of other investment to DG and feeders investment; $\alpha_{\mathrm{DG}}$ is the average annual cost coefficient of DG while $\alpha_{\mathrm{f}}$ is the average annual cost coefficient of feeders, they are related to the discount rate and service life; $P_{N \mathrm{DG} i(k)}, P_{E S S i(k)}$ and $c_{i}$ are the rated total installed capacity, the installed capacity of energy storage and the installation cost of unit capacity at the node $i ; c_{\mathrm{f}}$ is the investment cost of feeders; $X_{i \mathrm{DG}(k)}$ is the number of wind turbines installed on the node $i$; $P_{i \mathrm{DG}(k)}^{m, n}$ is the wind power at the node $i$ participated by energy storage in the $k$ th random fuzzy simulation.

In addition, the constraints should include equality constraints of node power, node voltage, branch power and random fuzzy chance constraints of backward power, wind power penetration, inequality constraints of DG amount, energy storage state and power capacity constraints.

$$
\left\{\begin{array}{l}
P_{i, \mathrm{DG}}-P_{i, \mathrm{~L}}=P_{i}^{\mathrm{inj}}(U, \theta) \\
Q_{i, \mathrm{DG}}-Q_{i, \mathrm{~L}}=Q_{i}^{\mathrm{inj}}(U, \theta) \\
\mathrm{Ch}\left\{U_{\min } \leq \tilde{U} \leq U_{\max }\right\}\left(\alpha_{u}\right) \geq \beta_{u} \\
\mathrm{Ch}\left\{\tilde{P}_{l} \leq P_{l \text { max }}\right\}\left(\alpha_{l}\right) \geq \beta_{l} \\
\mathrm{Ch}\left\{\tilde{\mathrm{P}}_{B} \geq 0\right\}\left(\alpha_{B}\right) \geq \beta_{B} \\
C P \leq C P_{\max } \\
0 \leq X_{i \mathrm{DG}} \leq X_{i \mathrm{DG}, \max } \\
S O C_{k}^{\min } \leq S O C_{k} \leq S O C_{k}^{\max } \\
0 \leq P_{c h a, t} \leq P_{c h a, \max } \\
0 \leq P_{d i s, t} \leq P_{d i s, \max } \\
0 \leq E_{b a t, i} \leq E_{b a t}^{\max }
\end{array}\right.
$$

and,

$$
C P=P_{\mathrm{DG}(k)} / P_{\mathrm{L}(k)}
$$

where, $P_{i, \mathrm{DG}}$ and $P_{i, \mathrm{~L}}$ are the DG active output and the active load at the node $i ; Q_{i, \mathrm{DG}}, Q_{i, \mathrm{~L}}$ stand for the DG reactive output and reactive load at the node $i ; P_{i}^{\mathrm{inj}}$, $Q_{i}^{\text {inj }}$ are the active and reactive at the node $i ; \alpha_{u}, \alpha_{l}, \alpha_{B}$ are the confidence levels of chance measurefor node voltage, branch power and forbidden backward power; $\beta_{u} 、 \beta_{l}, \beta_{B}$ are the lower limits of chance measure of node voltage, branch power and forbidden backward power; $C P$ is wind power penetration; $C P_{\max }$ is the maximum wind power penetration allowed; $X_{i \mathrm{DG} \text {, max }}$ is the maximum number of installed units allowed at nodes; $P_{\mathrm{DG}(k)}$ is annual wind output for the $k$ th simulation; $P_{\mathrm{L}(k)} \quad$ is the maximum load samples for the $k$ th simulation; $S O C_{k}^{\min }$ and $S O C_{k}^{\max }$ are the maximum and minimum charge state of the energy storage system; $P_{\text {cha,max }}$ and $P_{d i s, \max }$ are the maximum charge power allowed and maximum discharge power allowed of the battery system; $E_{b a t}^{\max }$ is the maximum capacity of storage system at a single node $i$. 


\subsection{The lower-level optimization model}

Based on the upper level optimization, the lower-level optimization is to optimize the wind power output in different periods. Its objective function is as follows:

\subsubsection{Objective function}

$$
\begin{gathered}
\min \overline{f_{d}} \\
f_{d}\left(X, \xi_{k}\right)=\omega_{\mathrm{DG}}^{\mathrm{cur}} \sum_{j=1}^{N_{\mathrm{DWG}}}\left(P_{\mathrm{DG} i(k)}^{m, n}+P_{\mathrm{ess} i(k)}^{m, n}\right)
\end{gathered}
$$

where, $\omega_{\mathrm{DG}}^{\mathrm{cur}}$ is the active reduction rate of DG, i.e. the proportion of active power reduction ; $P_{\mathrm{DG} i(k)}^{m, n}$ and $P_{\mathrm{essi}}^{m, n}(k)$ are the wind power and storage power at the node $i$ in the $n$th period of the season $m$ in the $k$ th random fuzzy simulation respectively.

\subsubsection{Constraint conditions}

The equality constraints are shown in Eq.(18).

$$
\left\{\begin{array}{l}
\left(1-\omega_{\mathrm{DG}}^{\mathrm{cur}}\right)\left(P_{i, \mathrm{DG}}+P_{i, \text { ess }}\right)-P_{i, \mathrm{~L}}=P_{i}^{\mathrm{inj}}(U, \theta) \\
\left(1-\omega_{\mathrm{DG}}^{\mathrm{cur}}\right) \tan \varphi_{\mathrm{DG}}\left(P_{i, \mathrm{DG}}+P_{i, \mathrm{ess}}\right)-Q_{i, \mathrm{~L}}=\mathrm{Q}_{i}^{\mathrm{inj}}(U, \theta)
\end{array}\right.
$$

The inequality constraints are shown in Eq.(19).

$$
\left\{\begin{array}{l}
\omega_{\mathrm{DG}, \text { min }}^{\text {cur }} \leq \omega_{\mathrm{DG}}^{\text {cur }} \leq \omega_{\mathrm{DG}, \text { max }}^{\text {cur }} \\
V_{\mathrm{OLTC}, \text { min }} \leq V_{\mathrm{OLTC}} \leq V_{\mathrm{OLTC}, \text { max }} \\
\cos \varphi_{\mathrm{DG}, \text { min }} \leq \cos \varphi_{\mathrm{DG}} \leq \cos \varphi_{\mathrm{DG}, \max }
\end{array}\right.
$$

Where, $\omega_{\mathrm{DG}, \max }^{\text {cur }}$ and $\omega_{\mathrm{DG}, \min }^{\text {cur }}$ are the upper and lower limits of DG active reduction rate; $V_{\text {OLTC,max }}$ and $V_{\text {OLTC,min }}$ are the upper and lower limits of secondary voltage of on-load tap changing transformer; $\cos \varphi_{\mathrm{DG}, \max }$ and $\cos \varphi_{\mathrm{DG}, \min }$ are the upper and lower limits of DG power factor.

\section{Solution of the model}

We combine random fuzzy simulation, forward and backward substitution method and genetic algorithm to solve the model. The process of random fuzzy modeling of distributed wind power output and static security index is metioned above. Mixed integer coding operator is used to solve the initial individual, and mixed integer mutation operator is used to solve the mutation individual. The number of wind turbines and energy storage configuration is set as the upper chromosome, and the three AM measures are set as the lower chromosome.

\section{Simulation and result analysis of example}

\subsection{Random fuzzy simulation of wind speed and static security index}

The DG output and active load obtained from timing simulation are as follows:

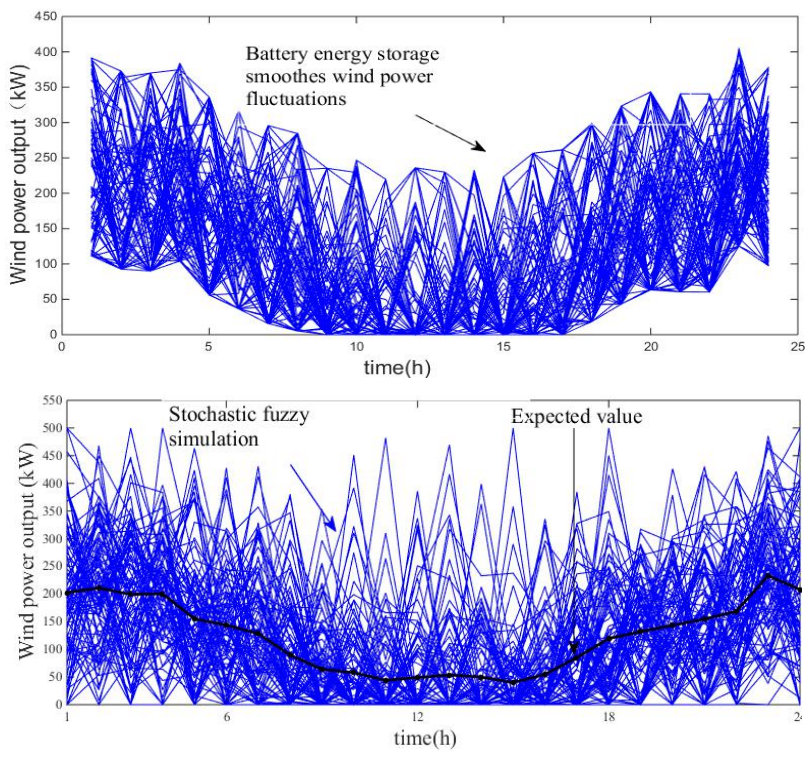

Figure 1. Wind power and load samples simulation diagram with scheduling

\subsection{Comparison of wind-storage cofiguration and cofiguration without energy storage}

,The results of wind-storage configuration and wind power only under fuzzy uncertain characteristics are analyzed with consistent parameters to study the effects of wind configuration in distribution network after considering the improvement of wind output fluctuation by energy storage. The results of comparative analysis are shown in Table 1.

Table 1. Comparison of the effects of uncertainty

\begin{tabular}{ccr}
\hline $\begin{array}{c}\text { Simulation } \\
\text { situation }\end{array}$ & $\begin{array}{c}\text { Wind-storage } \\
\text { configuration }\end{array}$ & $\begin{array}{c}\text { Wind } \\
\text { configuration } \\
\text { without stor }\end{array}$ \\
$\begin{array}{c}\text { number of wind } \\
\text { turbines at node } \\
(3,5,12)\end{array}$ & $7,3,4$ & $6,2,2$ \\
$\begin{array}{c}\text { energy storage } \\
\text { configuration }\end{array}$ & $491,213,366$ & 0 \\
$\begin{array}{c}\text { capacity[kW·h] } \\
\text { annual profit }\end{array}$ & 26430 & 27850 \\
$\begin{array}{c}\text { thousand yuan] } \\
\text { chance measure } \\
\text { under constraint } \\
\text { of node voltage } \\
\text { chance measure }\end{array}$ & 0.985 & 0.970 \\
\hline
\end{tabular}




\begin{tabular}{ccc}
$\begin{array}{c}\text { under constraint of } \\
\text { branch voltage } \\
\text { chance measure } \\
\text { under constraint of } \\
\text { backward voltage } \\
\text { if AM is applied } \\
\text { penetration of } \\
\text { wind power }\end{array}$ & 0.995 & 0.978 \\
$\begin{array}{c}\text { annual wind } \\
\text { output [MW·h] } \\
\text { annual load } \\
{[\mathrm{MW} \cdot \mathrm{h}]}\end{array}$ & 0.092 & yes \\
\hline
\end{tabular}

As is shown in Table 1, for one thing, the annual profit of wind-storage configuration decreased by $5.1 \%$, for the restrictions on optimization by the cost of energy storage, which result in a profits reduction. For another thing, the penetration of wind power increased from $7.1 \%$ to $9.2 \%$. Through the analysis of the expressions, the load of the two models is nearly equal. The main reason causing the increase of penetration is that, the wind will be abandoned without considering the limitation of the maximum fluctuation of the wind-storage system when in a sharp fluctuation period. The wind-storage combined system can use the energy storage to improve the uncertainty by peak-load shifting. Hence, the supply and the penetration of wind power are increased.

\subsection{Influence of confidence level on configuration}

In random fuzzy chance constrained programming, the confidence level represents the trustworthiness of results. Setting the confidence level of 0.95 indicates that the random fuzzy chance constrained scheme is established when the lower limit of the chance measure, satisfying the static security index, is greater than $95 \%$. The random fuzzy expectations of profit are shown in Table 2 when the confidence level of static security indicators is respectively $0.90,0.92,0.94,0.96$ and 0.98 .

Table 2. Comparison between the results of bi-level programming under different confidence level

\begin{tabular}{cccccc}
\hline $\begin{array}{c}\text { confidence level } \\
\text { number of wind }\end{array}$ & $\mathbf{0 . 9 0}$ & $\mathbf{0 . 9 2}$ & $\mathbf{0 . 9 4}$ & $\mathbf{0 . 9 6}$ & $\mathbf{0 . 9 8}$ \\
$\begin{array}{c}\text { turbines at node } \\
(3,5,12)\end{array}$ & $8,4,4$ & $8,3,4$ & $7,3,4$ & $7,3,4$ & $7,3,3$ \\
$\begin{array}{c}\text { energy storage } \\
\text { configuration }\end{array}$ & 491, & 495, & 539, & 556, & 550, \\
$\begin{array}{c}\text { capacity[kW·h] } \\
\text { annual }\end{array}$ & 306 & 300 & 301 & 306 & 246 \\
$\begin{array}{c}\text { pofit[thousand } \\
\text { yuan] }\end{array}$ & 27840 & 27180 & 26700 & 26130 & 25390 \\
& & & & &
\end{tabular}

\begin{tabular}{cccccc}
$\begin{array}{c}\text { net loss[thousand } \\
\begin{array}{c}\text { yuan] } \\
\text { environmental }\end{array}\end{array}$ & 1000 & 980 & 950 & 960 & 940 \\
$\begin{array}{c}\text { improvement } \\
\text { profit[10000 yuan] } \\
\text { annual active }\end{array}$ & 1920 & 1790 & 1680 & 1680 & 1560 \\
$\begin{array}{c}\text { reduction[MW·h] } \\
\text { penetration of } \\
\text { wind power }\end{array}$ & 0.108 & 0.099 & 0.092 & 0.089 & 0.087 \\
$\begin{array}{c}\text { node voltage } \\
\text { chance measure } \\
\text { branch power } \\
\text { chance measure }\end{array}$ & 0.945 & 0.953 & 0.968 & 0.985 & 0.991 \\
$\begin{array}{c}\text { backward power } \\
\text { chance measure }\end{array}$ & 0.913 & 0.968 & 0.975 & 0.987 & 0.990 \\
\hline
\end{tabular}

As is shown in Table 2, the higher the confidence level is, the lower the annual profit and the wind power penetration are. Therefore, in the proposed optimal configuration of distributed power based on random fuzzy chance constrained programming, the planners can achieve the maximum annual profit by setting different confidence levels of chance measure within an allowed risk range according to the requirement.

\section{Conclusion}

In this paper, A random fuzzy chance constrained bilevel programming scheme for distributed wind power and energy storage combined system is proposed. The results can be summarized as follows:

i) The scheme comprehensively takes the objective temporal characteristics of the wind speed and the random fuzzy uncertainties into consideration and describes the static security index with random fuzzy chance measure, which will enrich uncertain information of configuration.

ii) The capacity of wind turbine and energy storage is planned jointly in the proposed scheme, for improving the fluctuation characteristics of wind power output. The scheme is able to fully consider the peak load shifting of energy storage to improve the operation of distribution networks. The capacity allocation scheme of distributed wind has stronger adaptability under the precondition of improving DG acceptance.

iii) The scheme considers different confidence levels of the system static security index, provides economic benefits evaluation such as annual profit under the premise of feasibility, which allows planners allocate distributed wind capacity flexibly considering both technical and economic perspectives.

\section{References}

1. L. Li, W. Tang, M. Bai, et al. Multi-objective locating and sizing of distributed generators based on timesequence characteristics[J]. Automation of Electric Power Systems, 2013, 37(3):58-63. 
2. A. Soroudi, R. Caire, N. Hadjsaid, et al. Probabilistic dynamic multi-objective model for renewable and nonrenewable distributed generation planning[J]. IET Generation, Transmission\& Distribution, 2011,5 (11):1173-1182.

3. S. Zhang, K. Li, H. Cheng, et al. Optimal siting and sizing of intermittent distributed generator considering correlations [J]. Automation of Electric Power Systems, 2015, 39(8):53-58.

4. M R. Haghifam, H. Falaghi, O P. Malik. Risk-based distributed generation placement $[\mathrm{J}]$. Iet Generation Transmission \& Distribution, 2008, 2(2):252-260.

5. 5. X. Han, J. LI, J. Wen. Optimization for Robust Energy Storage Allocation in Power System With Multiple Wind Farms Integrated[J]. Transactions of China Electrotechnical Society, 2015, 35(09):2120-2127. 